\title{
Site-specific lipidation enhances IFITM3 membrane interactions and antiviral activity
}

\section{Supplementary Information}

Emma Garst ${ }^{1,2 \dagger}$, Hwayoung Lee ${ }^{3 \dagger}$, Tandrila Das ${ }^{1,2 \dagger}$, Shibani Bhattacharya ${ }^{4}$, Avital Percher ${ }^{1}$, Rafal Wiewiora ${ }^{2,5}$, Isaac Witte ${ }^{1}$, Yumeng $\mathrm{Li}^{6}$, Tao Peng ${ }^{1,6}$, Wonpil Im³, Howard C. Hang ${ }^{1,7 *}$

${ }^{1}$ Laboratory of Chemical Biology and Microbial Pathogenesis, The Rockefeller University, New York, New York 10065, United States.

${ }^{2}$ Tri-Institutional Ph.D. Program in Chemical Biology, New York, NY 10065, United States.

${ }^{3}$ Department of Biological Sciences, Chemistry, and Bioengineering, Lehigh University, Bethlehem, PA 18015, United States.

${ }^{4}$ New York Structural Biology Center, New York, NY 10027, United States.

${ }^{5}$ Memorial Sloan Kettering Cancer Center, New York, NY 10065, United States.

${ }^{6}$ State Key Laboratory of Chemical Oncogenomics, School of Chemical Biology and Biotechnology, Peking University Shenzhen Graduate School, Shenzhen 518055, China.

${ }^{7}$ Departments of Immunology and Microbiology and Chemistry, Scripps Research, La Jolla, CA 92037, United States.

†These authors contributed equally.

*Correspondence: hhang@scripps.edu 


\section{SUPPLEMENTARY FIGURES}

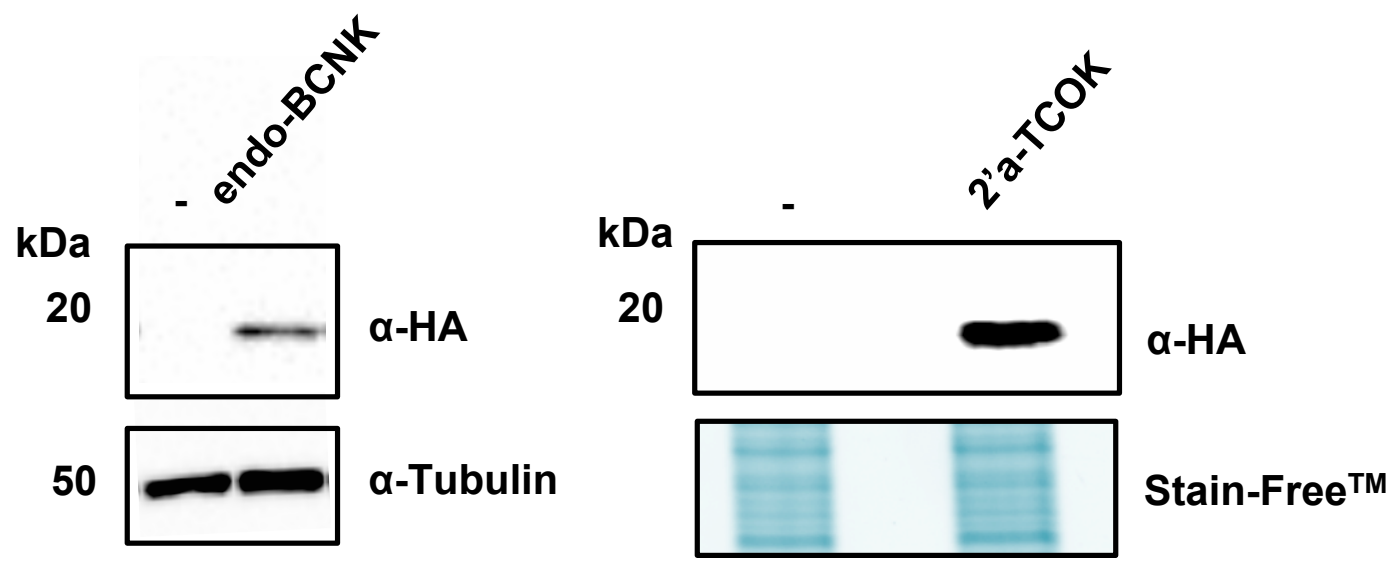

Supplementary Figure 1. Western blot analysis of HA-IFITM3-Cys72TAG expression with different unnatural amino acids (UAAs). HEK293T cells were transfected with plasmids encoding an aminoacyl-tRNA synthetase/tRNA pair MmPyIRS-AF (Y306A, Y384F)/Pyl-tRNA and HA-IFITM3-Cys72TAG in the absence or presence of different UAAs. Anti-HA blot shows efficient genetic code expansion for HAIFITM3-C72TAG expression with different UAAs. Anti-Tubulin blot or Stain-Free ${ }^{\mathrm{TM}}$ gel (Bio-Rad) is shown as protein loading control. 


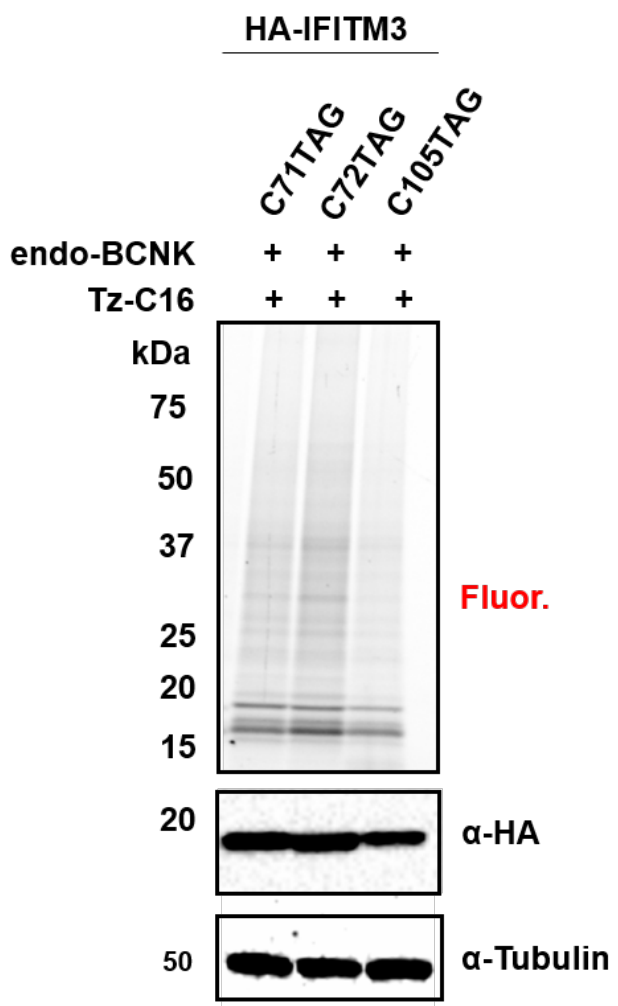

Supplementary Figure 2. In-gel fluorescence profiling of tetrazine ligation efficiency at different Cys positions in IFITM3 in cells. HEK293T cells were transfected with plasmids encoding an aminoacyl-tRNA synthetase/tRNA pair MmPyIRS-AF (Y306A, Y384F)/Pyl-tRNA and HA-IFITM3-Cys71TAG or Cys72TAG or Cys105TAG in the presence of endo-BCNK, after which cells were treated with fatty acyl tetrazine Tz-6. The cell lysates were further reacted with azide-rhodamine for in-gel fluorescence profiling of chemically lipidated IFITM3. An anti-HA blot revealed similar IFITM3 expression levels in each condition. 
A

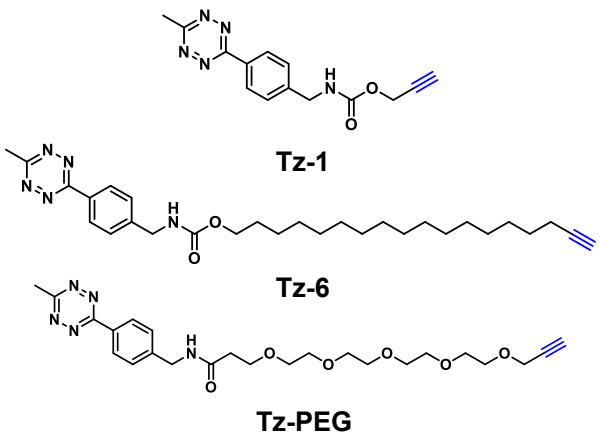

B

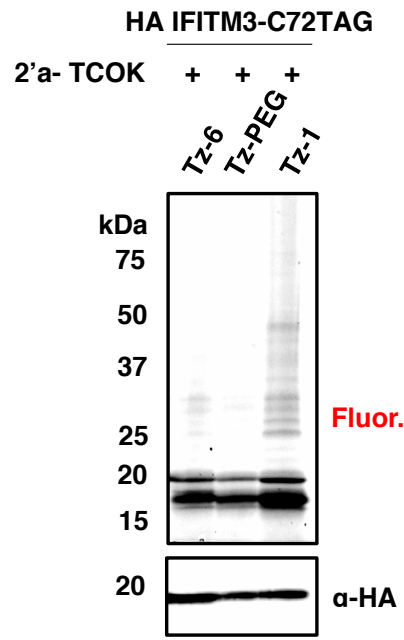

C

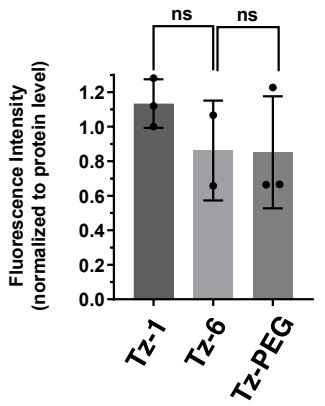

Supplementary Figure 3. In-gel fluorescence profiling of tetrazine ligation efficiency with different tetrazine derivatives in cells. A) Structures of the different tetrazine derivatives used for tetrazine ligation to UAAs. B) HEK293T cells were transfected with plasmids encoding an aminoacyl-tRNA synthetase/tRNA pair MmPyIRS-AF (Y306A, Y384F)/Pyl-tRNA and HA-IFITM3-Cys72TAG in the presence of 2'aTCOK, after which cells were treated with different tetrazine analogs. The cell lysates were further reacted with azide-rhodamine for in-gel fluorescence profiling of chemically modified IFITM3. Anti-HA blot shows IFITM3 expression level in each condition. C) Quantification of efficiency of in-cell tetrazine ligation reaction. Data represents mean \pm S.D. for three independent experiments. Data were analyzed by unpaired Student's ttest (ns $p>0.05$ ). 
HeLa WT

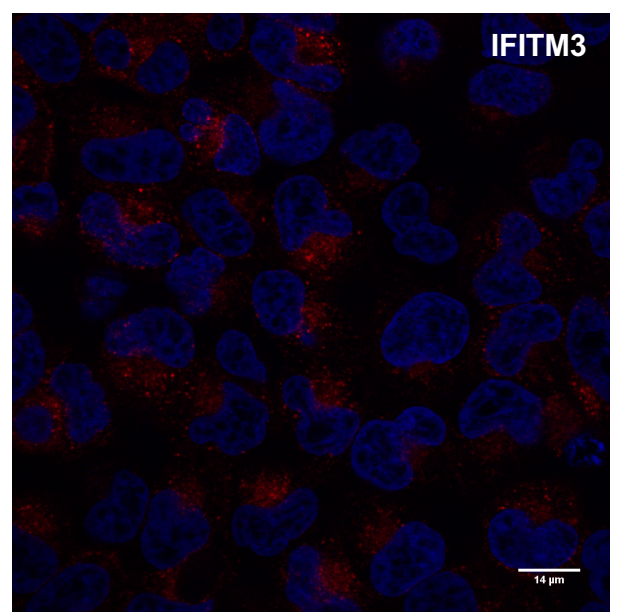

HeLa IFITM2/3 KO

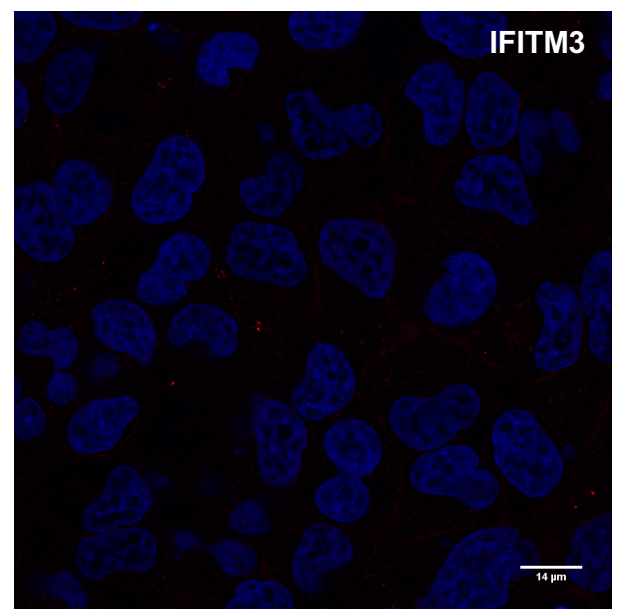

Supplemental Figure 4. Immunofluorescence imaging for characterization of HeLa WT and HeLa IFITM2/3 KO cells. HeLa WT and HeLa IFITM2/3 KO cells were stained with anti-IFITM3 (red) antibody to demonstrate IFITM3 protein levels. DAPI (blue) was used as a nuclear stain. 

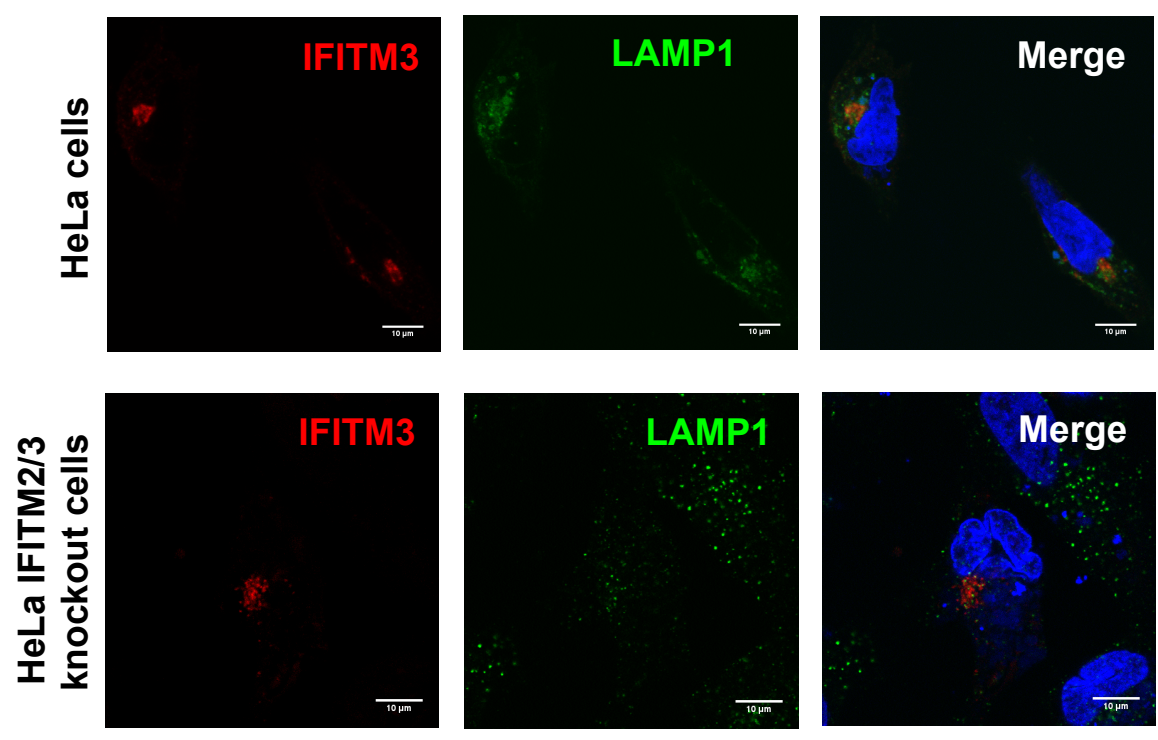
HA IFITM3-Cys72TAG- endo-BCNK
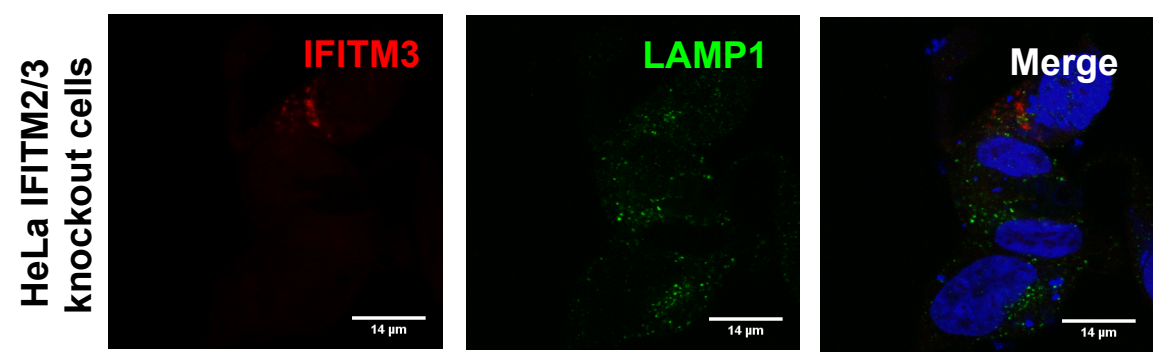

HA IFITM3-Cys72TAGendo-BCNK-Tz-6

Supplementary Figure 5. Subcellular localization of chemically modified HAIFITM3-Cys72TAG. IFITM 2/3 KO HeLa cells were transfected with plasmids encoding an aminoacyl-tRNA synthetase/tRNA pair Mm-PyIRS-AF (Y306A, Y384F)/Pyl-tRNA and HA-IFITM3-Cys72TAG in the presence of endo-BCNK, after which cells were treated with tetrazine analogue Tz-6. Cells were stained with anti-IFITM3 antibody, anti-LAMP1 antibody, and DAPI for nucleus. Top row, localization of endogenous IFITM3 (red) and LAMP1 (green) in HeLa cells; middle row, LAMP1 (green) and HA-IFITM3 with endoBCNK at Cys72 (red) expressed in HeLa IFITM2/3 knockout cells; bottom row, LAMP1 (green) and HA-IFITM3 chemically lipidated with Tz-6 in HeLa IFITM2/3 knockout cells. 
A

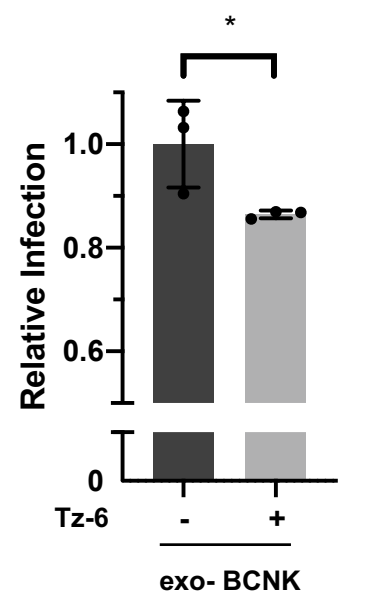

HA-IFITM3-Cys71TAG
B

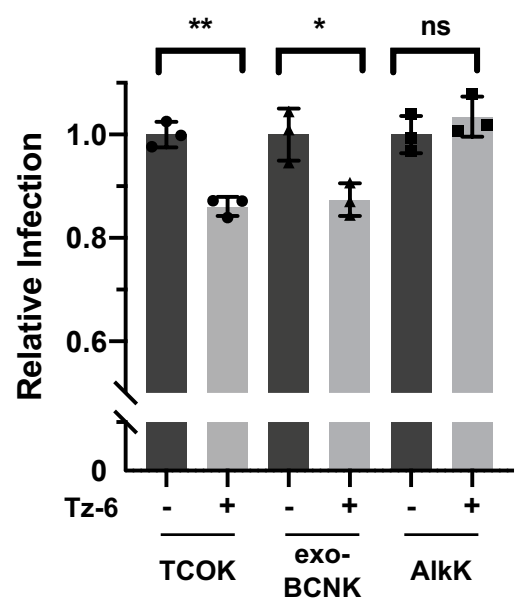

HA-IFITM3-Cys72TAG

Supplementary Figure 6. Antiviral activity of chemically lipidated HA-IFITM3. Quantification of influenza A virus (IAV) infection assay of HA-IFITM3 Cys71TAG and Cys72TAG-expressing cells with and without Tz-6 ligation. Cells expressing HA-IFITM3Cys71TAG or Cys72TAG with exo-BCNK were treated with fatty acyl tetrazine analog Tz-6, then infected with IAV. Virus nucleoprotein (NP) and HA-IFITM3 protein levels were examined by flow cytometry using anti-NP and anti-IFITM3 staining, respectively. Transfected cells expressing IFITM3 were gated and analyzed for the percentage of infection. Relative infection for IFITM3 expressing cells was calculated for with and without Tz-6-treated samples. Data represents mean \pm S.D. for three independent experiments. Data were analyzed by unpaired Student's t-test (ns $p>0.05,{ }^{*} p<0.05$, $\left.{ }^{* *} p<0.005\right)$. 


$\begin{array}{rlccccccccc}\text { Time (h) } & 16 & 16 & 16 & 16 & 16 & 16 & 16 & 4 & 2 & 4 \\ \left.\text { Temp ( }{ }^{\circ} \mathrm{C}\right) & 4 & 4 & 4 & 4 & 4 & 10 & 10 & 15 & 15 & 15 \\ \text { TCEP (mM) } & 1 & 1 & 1 & 1 & 5 & 1 & 1 & 1 & 1 & 1 \\ \text { mPalm (mM) } & 0 & 0.1 & 0.5 & 1 & 0.5 & 0 & 0.5 & 0 & 0.5 & 0.5 \\ 15 \mathrm{KDa} & \end{array}$

Supplementary Figure 7. Optimization of Cys72-IFITM3 maleimide-palmitate coupling reaction. A number of conditions were tested for the maleimide-palmitate (mPalm) coupling reaction, such as time, temperature, TCEP concentration, and $\mathrm{mPalm}$ concentration. SUMO was present throughout the reaction and acted as a loading control. Successful coupling was monitored by a mass shift on an SDS PAGE gel. At $4^{\circ} \mathrm{C}$ and $10^{\circ} \mathrm{C}$, the maleimide coupling reaction did not go to completion after overnight coincubation with Cys72-IFITM3, as shown by a clear double band. Neither increasing the mPalm concentration nor increasing the TCEP concentration pushed the reaction to completion. However, at $15^{\circ} \mathrm{C}$ the reaction went to completion in 2 hours. 


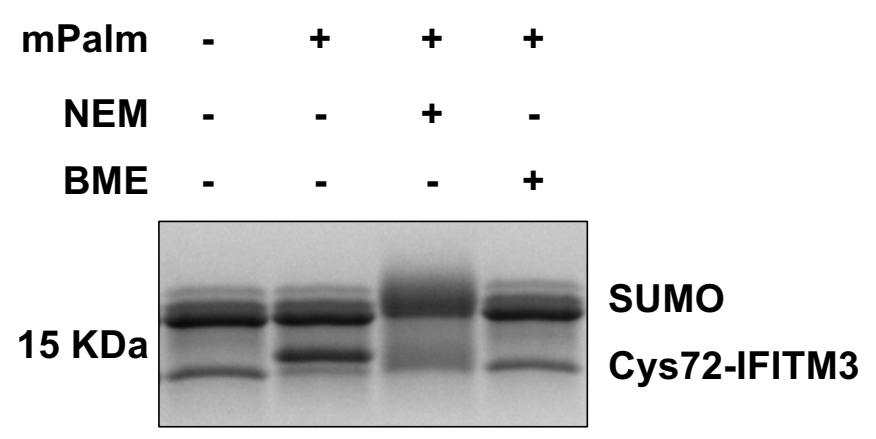

Supplementary Figure 8. Maleimide-palmitate coupling with Cys72-IFITM3 can be blocked or quenched by $\mathbf{N}$-ethyl maleimide (NEM) or $\beta$-mercaptoethanol (BME) respectively. In brief, to test the ability of NEM and BME to block the mPalm-IFITM3 coupling reaction, Cys72-IFITM3 was pre-incubated with $20 \mathrm{mM}$ NEM for 30 minutes prior to $\mathrm{mPalm}$ coupling (NEM+ condition), or mPalm was preincubated with $5 \mathrm{mM} \mathrm{BME}$ for 30 minutes before the Cys72-IFITM3 coupling reaction (BME+ condition). Maleimidepalmitate coupling to Cys72-IFITM3 was monitored via mass shift in an Coomassie stained SDS PAGE gel. SUMO was present throughout the reaction and confirms the SDS PAGE gel is not warped. While a clear band shift was seen when Cys72-IFITM3 was coincubated directly with $\mathrm{mPalm}$ as previously described, coincubation with NEM or BME appeared to abrogate the effect. 


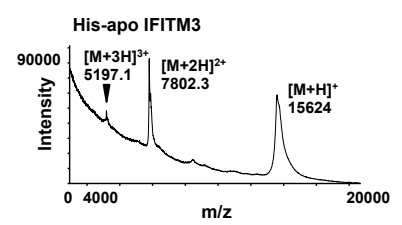

\begin{tabular}{|l|l|l|l|}
\hline & \multicolumn{1}{|c|}{$[\mathbf{M + H}]^{+}$} & \multicolumn{1}{c|}{$[\mathrm{M}+\mathbf{2 H}]^{2+}$} & \multicolumn{1}{c|}{$[\mathrm{M+3H}]^{3+}$} \\
\hline Expected mass (m/z) & 15765 & 7883.5 & 5255.0 \\
\hline Observed mass (m/z) & 15624 & 7802.3 & 5197.1 \\
\hline Difference (Da) & -141 & -81.2 & -57.9 \\
\hline
\end{tabular}

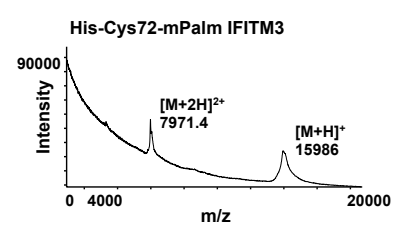

\begin{tabular}{|l|l|l|l|}
\hline & \multicolumn{1}{|c|}{$[\mathbf{M + H}]^{+}$} & \multicolumn{1}{c|}{$[\mathbf{M + 2 H}]^{2+}$} & \multicolumn{1}{c|}{$[\mathbf{M + 3 H}]^{3+}$} \\
\hline Expected mass (m/z) & 16119 & 8060.3 & 5373.9 \\
\hline Observed mass (m/z) & 15986 & 7971.4 & N/A \\
\hline Difference (Da) & -133 & -88.9 & N/A \\
\hline
\end{tabular}

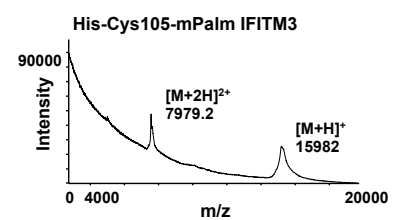

\begin{tabular}{|l|l|l|l|}
\hline & \multicolumn{1}{|c|}{$[\mathbf{M + H}]^{+}$} & \multicolumn{1}{c|}{$[\mathbf{M + 2 H}]^{2+}$} & \multicolumn{1}{c|}{$[\mathbf{M + 3 H}]^{3+}$} \\
\hline Expected mass (m/z) & 16119 & 8060.3 & 5373.9 \\
\hline Observed mass (m/z) & 15982 & 7979.2 & N/A \\
\hline Difference (Da) & -137 & -81.1 & N/A \\
\hline
\end{tabular}

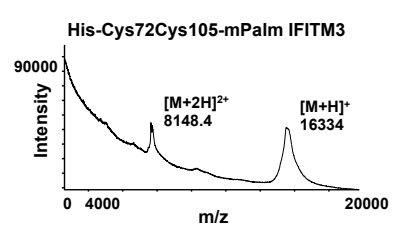

\begin{tabular}{|l|l|l|l|}
\hline & \multicolumn{1}{|c|}{$[\mathrm{M}+\mathrm{H}]^{+}$} & \multicolumn{1}{c|}{$[\mathrm{M+2H}]^{2+}$} & \multicolumn{1}{c|}{$[\mathrm{M+3H}]^{3+}$} \\
\hline Expected mass (m/z) & 16472 & 8237.1 & 5491.7 \\
\hline Observed mass (m/z) & 16334 & 8148.4 & N/A \\
\hline Difference $(\mathrm{Da})$ & -138 & -88.7 & N/A \\
\hline
\end{tabular}

Supplementary Figure 9. MALDI quantification of $\mathrm{His}_{6}$-apo IFITM3 and panel of His $_{6}$-IFITM3 lipidated variants. The observed and expected masses of each His6IFITM3 variant were compared in order to confirm the efficacy of the mPalm coupling reaction. Comparison between spectra reveals a $362 \mathrm{Da}$ increase in mass for His 6 Cys72-mPalm-IFITM3 and $358 \mathrm{Da}$ increase in mass for His 6 -Cys105-mPalm-IFITM3 when compared to unlipidated IFITM3 (His 6 -apo-IFITM3). The dually lipidated IFITM3 construct (His6-Cys72Cys105-mPalm-IFITM3) has a mass increase of 710 Da over the unmodified construct. Each construct had a net 130 - $180 \mathrm{Da}$ loss in mass when compared to the expected molecular weight, indicating the loss of a terminal Met during expression or purification. 


\section{Apo IFITM3}

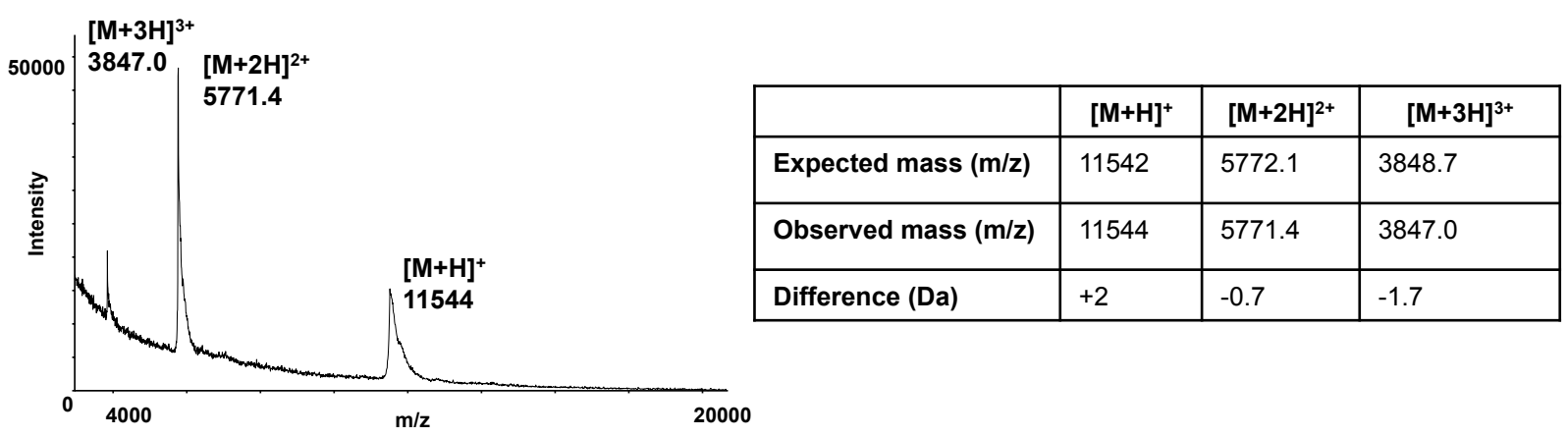

\section{Cys72-mPalm IFITM3}

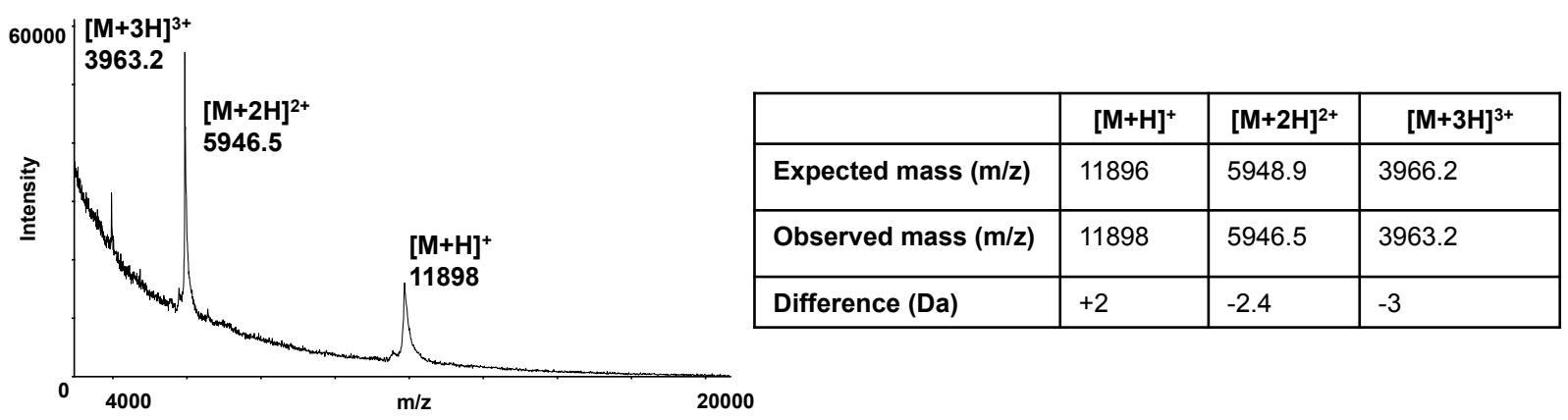

Supplementary Figure 10. MALDI analysis of apo 1-106 IFITM3 and Cys72-mPalm 1-106 IFITM3. MALDI spectra of apo 1-106 IFITM3 and Cys72-mPalm 1-106 IFITM3 were collected in linear, delayed extraction mode a 2 ns sampling rate and $0.75-1 \mu s$ delay. Samples were calibrated internally with a horse myoglobin protein standard. The observed and expected masses of the apo 1-106 and Cys72-mPalm 1-106 IFITM3 variants were compared in order to confirm the efficacy of the mPalm coupling reaction. Comparison between spectra revealed a $354 \mathrm{Da}$ increase in mass, indicating the addition of one mPalm group. The expected and observed masses for both constructs were in accordance, with less than $a \pm 3$ Da difference for each peak. 

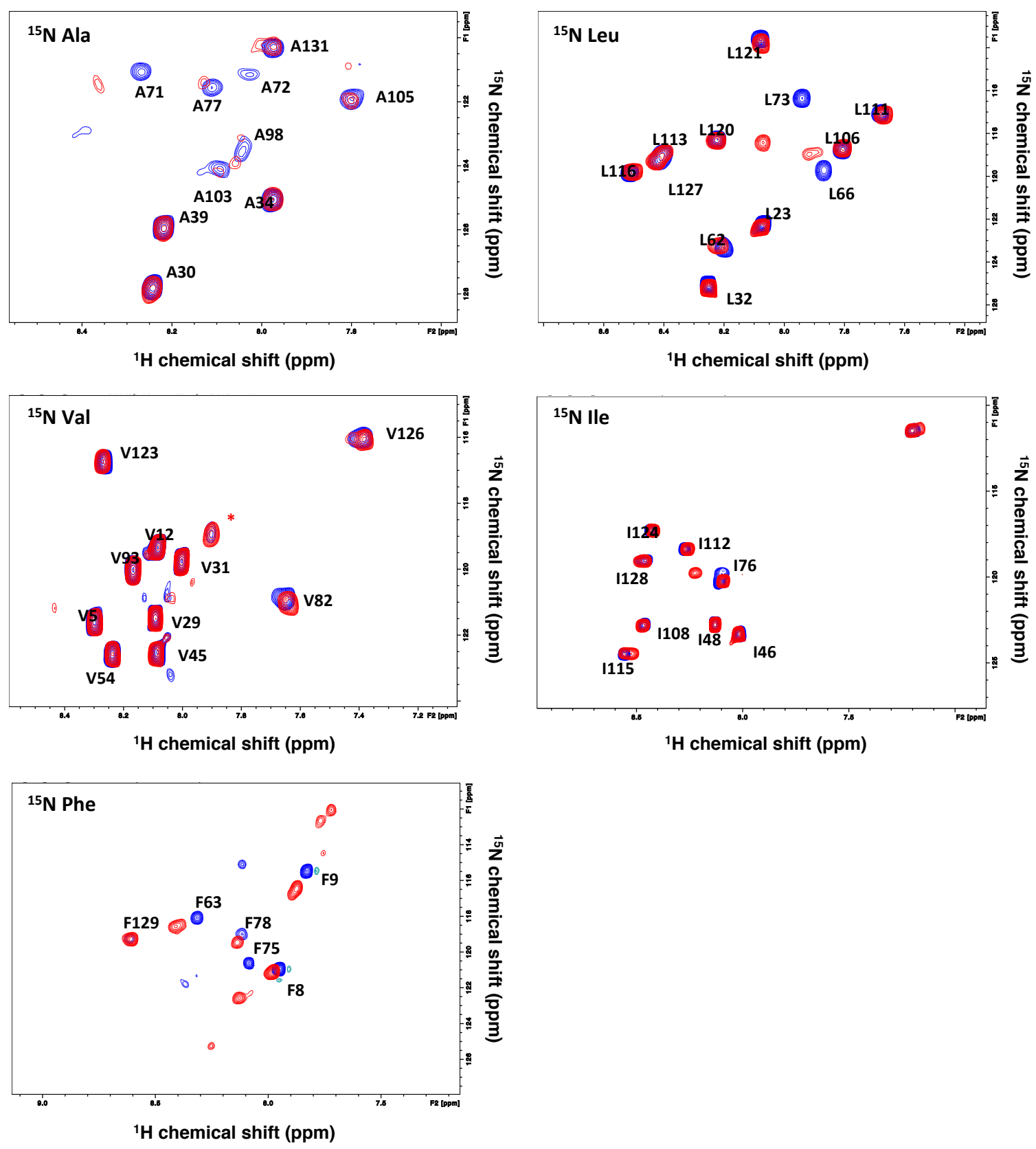

Supplementary Figure $11 .{ }^{15} \mathrm{~N}$ specific labeling of His 6 -apo and His 6 -Cys-mPalm IFITM3 for backbone assignment. ${ }^{1} \mathrm{H}-{ }^{15} \mathrm{~N}$ TROSY spectra were collected of ${ }^{15} \mathrm{~N}$ -

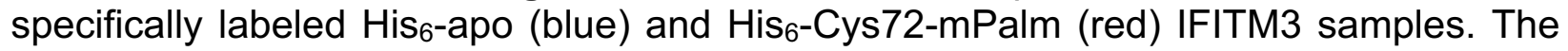
residues used in specific labeling were (clockwise from top left): ${ }^{15} \mathrm{~N} \mathrm{Ala},{ }^{15} \mathrm{~N}$ Leu, ${ }^{15} \mathrm{~N}$ lle, ${ }^{15} \mathrm{~N}$ Phe, and ${ }^{15} \mathrm{~N}$ Val. 


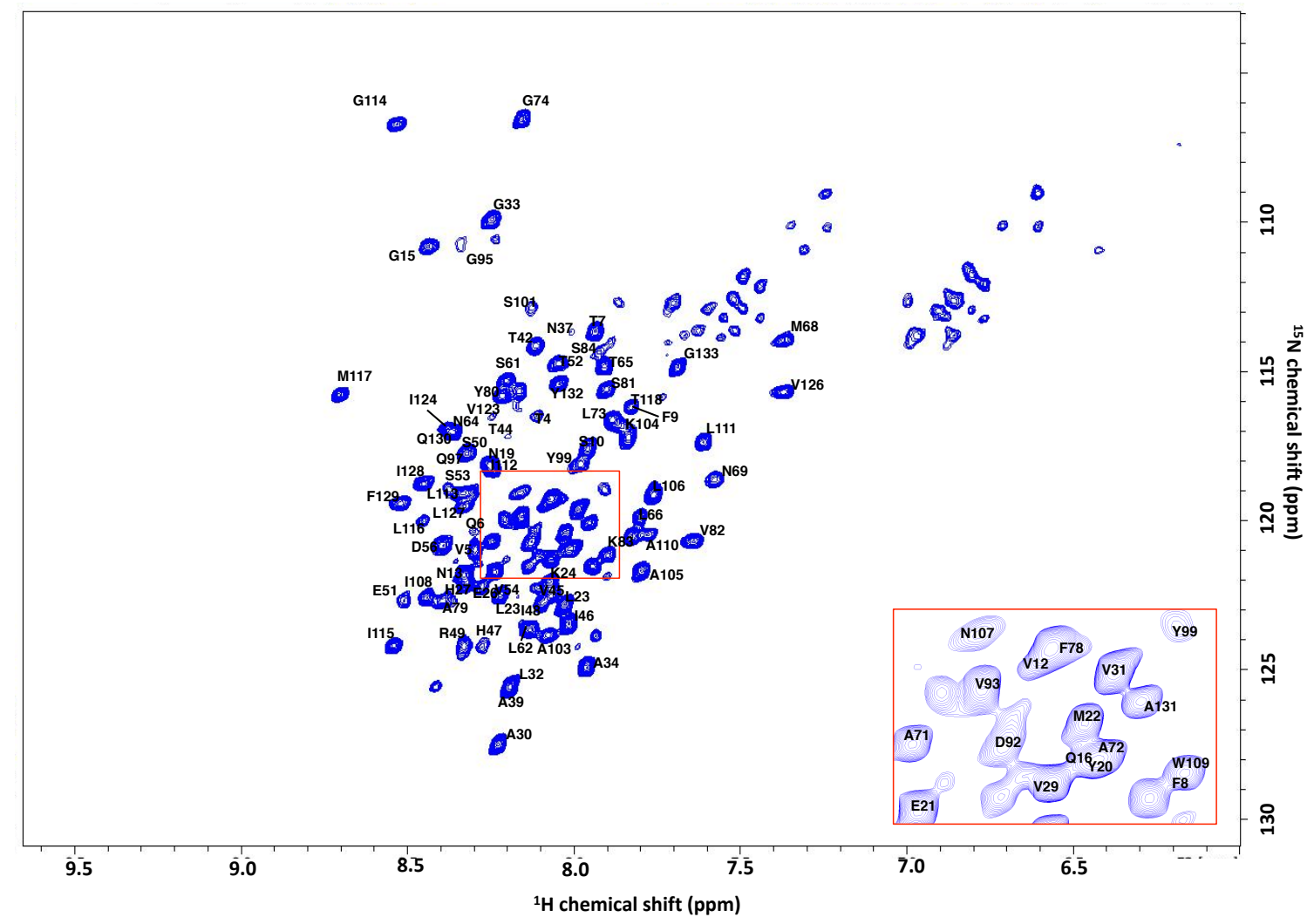

Supplementary Figure 12. Backbone resonance assignment of His6-apo IFITM3 overlayed on ${ }^{1} \mathrm{H}-{ }^{15} \mathrm{~N}$ TROSY spectra. All His 6 -apo IFITM3 assignment experiments were conducted on ${ }^{2} \mathrm{H}^{13} \mathrm{~N}^{15} \mathrm{C}$ labeled His 6 -apo IFITM3 at $35^{\circ} \mathrm{C}$ in $25 \mathrm{mM}$ HEPES pH 7 , $150 \mathrm{mM} \mathrm{KCl}$, and $0.5 \%$ DPC. Residue assignment was achieved via the following 3D experiments: $15 \mathrm{~N}-\mathrm{HSQC}$, HNCA, HNCO, HNCACB, HNCOCA, HNCOCACB, and HNCACO (see supplementary methods). 


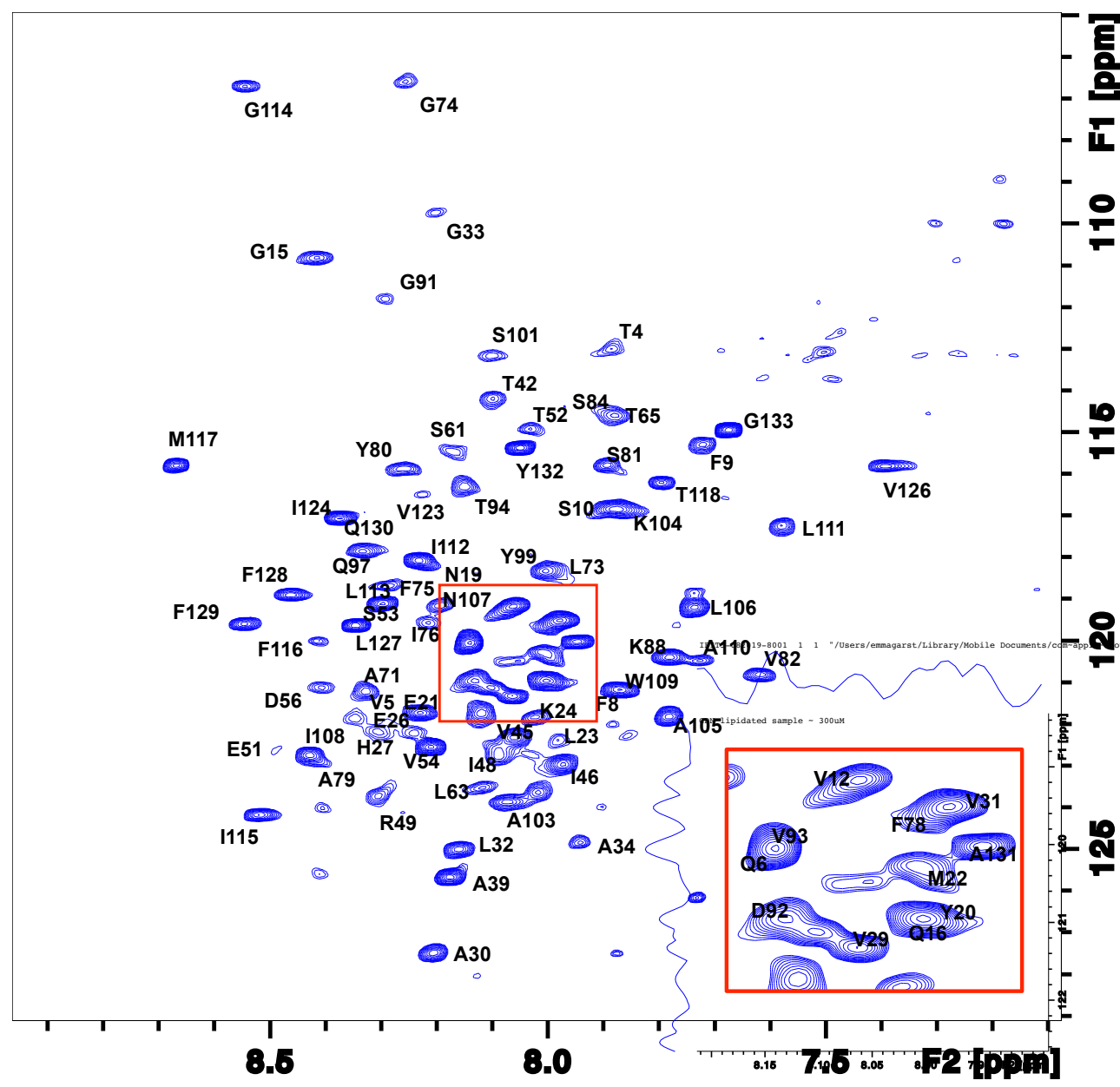

Supplementary Figure 13. Backbone resonance assignment of $\mathrm{His}_{6}-\mathrm{Cys}_{\mathbf{2}}$ 72-mPalm IFITM3 overlayed on ${ }^{1} \mathrm{H}-{ }^{15} \mathrm{~N}$ TROSY spectra. All His6-Cys72-mPalm IFITM3 assignment experiments were conducted on ${ }^{2} \mathrm{H}^{13} \mathrm{~N}^{15} \mathrm{C}$ labeled His6-apo IFITM3 at $35^{\circ} \mathrm{C}$ in $25 \mathrm{mM}$ HEPES $\mathrm{pH} 7,150 \mathrm{mM} \mathrm{KCl}$, and $0.5 \%$ DPC. Residue assignment was achieved via the following 3D experiments: 15N-HSQC, HNCA, HNCO, HNCACB, HNCOCA, HNCOCACB, and HNCACO (see supplementary methods). 
A

His apo IFITM3

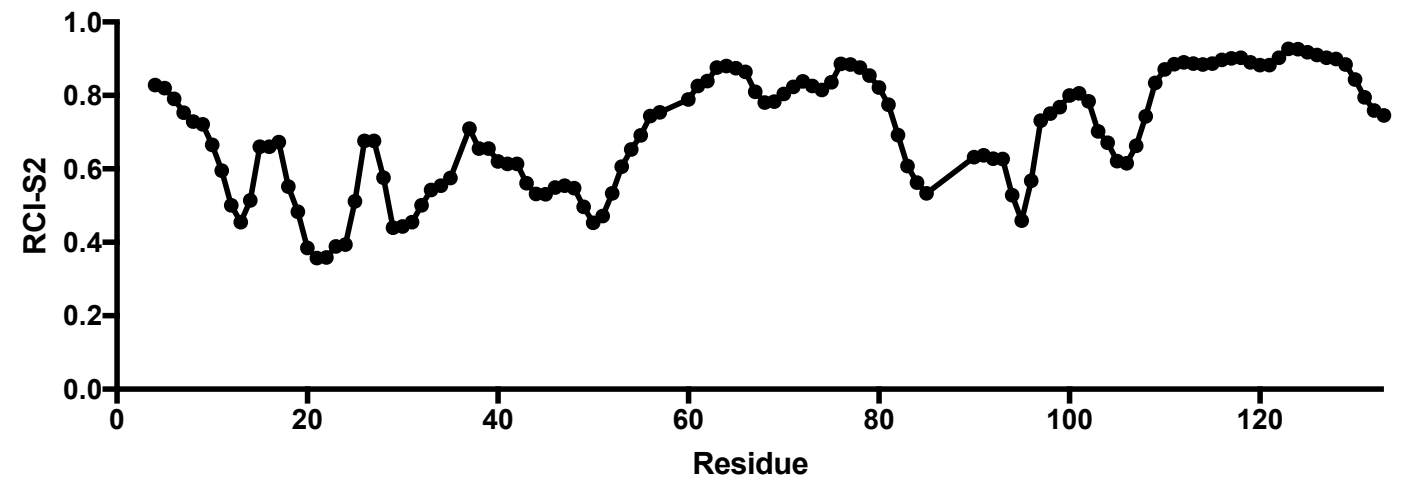

B

His Cys72-mPalm IFITM3

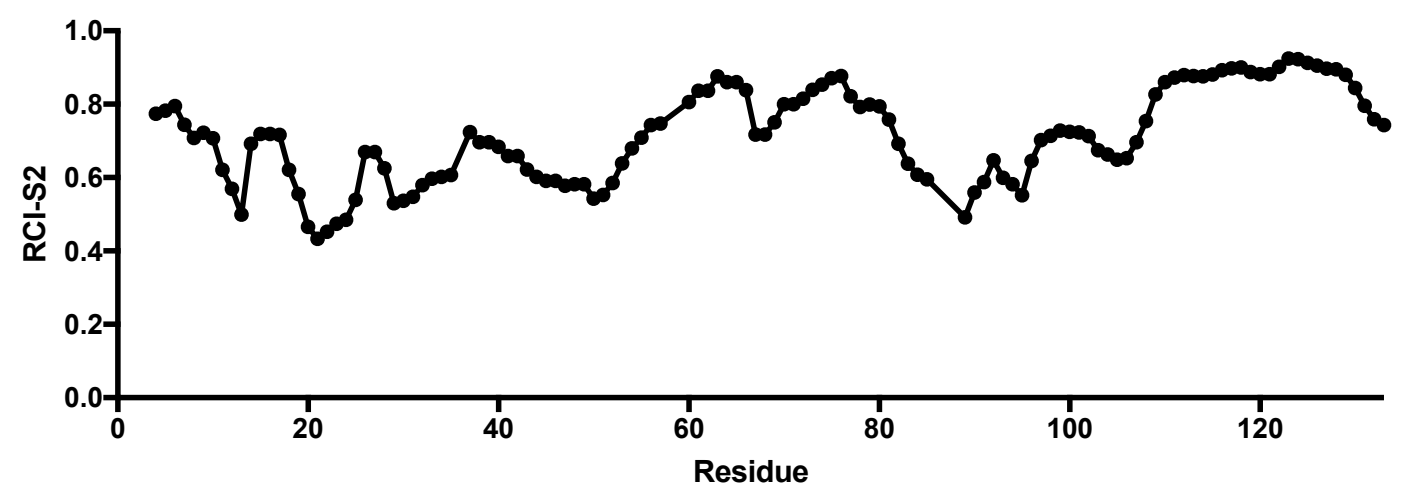

Supplementary Figure 14. RCI analysis of His 6 -apo IFITM3 and His 6 -Cys72-mPalm IFITM3. The random coil index predicted order parameter S2 was calculated from protein chemical shifts determined via backbone assignment of His $6^{-a p o}$ and $\mathrm{His}_{6}{ }^{-}$ Cys72-mPalm IFITM3. The calculation was preformed by the web-based TALOS+ analysis program ${ }^{1}$ as described in Berjanskii et al. ${ }^{2}$ 


\section{MATERIALS AND METHODS}

\section{Cell culture and reagents}

HEK293T cells were purchased from ATCC and cultured in Dulbecco's Modified Eagle's Medium (DMEM, high glucose; Gibco) supplemented with $10 \%$ fetal bovine serum (FBS; VWR). Influenza A/PR/8/34 (H1N1) (10100374) was from Charles River Laboratories. Influenza A virus nucleoprotein antibody [AA5H] (ab20343) was from Abcam. Alexa-Fluor 647 Antibody Labeling Kit (A20186) was ordered from Life Technologies. Anti-HA antibody conjugated to Alexa-Fluor 594 was purchased from Life Technologies. Anti-HA HRP-conjugated antibody (3F10) was ordered from Roche. AntiIFITM3 antibody (11714-1-AP) was purchased from Proteintech. Anti-rabbit IgG HRPconjugated antibody was ordered from Cell Signaling Technology. Anti-Tubulin HRPconjugated antibody $(11 \mathrm{H} 10)$ was also purchased from Cell Signaling Technology. Goat anti-rabbit IgG secondary antibody conjugated to Alexa-Fluor 647 (A27040) was ordered from Life Technologies.

\section{Plasmid transfection}

pCMV-Mm-PyIRS-WT plasmid was kindly provided by Professor Peng R. Chen at Peking University (Zhang et al., 2011). pCMV-FLAG-Mm-PyIRS-AF (Y306A and Y384F double mutant of wild-type PyIRS) were generated in the lab by site-directed mutagenesis of pCMV-Mm-PyIRS-WT and introducing an in-frame N-terminal FLAG tag. Human IFITM3 cDNA was purchased from Open Biosystems and PCR cloned into pCMV-HA vector (Clontech). All mutants of IFITM3 were generated by using QuikChange II Site-Directed Mutagenesis Kit (Agilent Technologies, \#200523). Lipofectamine 3000 from Thermo Scientific was used for transfection of HEK293T cells.

\section{Chemical lipidation of IFITM3}

HEK293T cells were seeded on 6-well plates and cultured overnight. The next day cells were co-transfected with the plasmid encoding aminoacyl-tRNA synthetase/tRNA pair Mm-PyIRS-AF (Y306A, Y384F)/Pyl-tRNA $(0.5 \mu \mathrm{g})$ and HA-IFITM3-Cys72TAG (0.5 $\mu \mathrm{g})$ using $3 \mu \mathrm{L}$ Lipofectamine 3000 in complete cell growth media containing UAAs (100 $\mu \mathrm{M})$ for $16 \mathrm{~h}$. Then cells were treated with tetrazine-lipid $(20 \mu \mathrm{M})$ for $2 \mathrm{~h}$. Cells were lysed with 4\% SDS lysis buffer (4\% SDS, $150 \mathrm{mM} \mathrm{NaCl}, 50 \mathrm{mM}$ triethanolamine $\mathrm{pH}$ 7.4, Roche protease inhibitor, benzonase). Protein concentrations were determined by the $\mathrm{BCA}$ assay (Pierce). Protein concentration of cell lysate was normalized to $1.6 \mathrm{mg} / \mathrm{ml}$.

\section{Fluorophore labeling of chemically lipidated IFITM3}

$45 \mu \mathrm{l}$ of the cell lysate was treated with $5 \mu \mathrm{l}$ of CuAAC reactant solution $(0.5 \mu \mathrm{l}$ of $10 \mathrm{mM}$ azido-rhodamine (final concentration $100 \mu \mathrm{M}$ ), $1 \mu \mathrm{l}$ of $50 \mathrm{mM}$ freshly prepared CuSO $_{4} \cdot 5 \mathrm{H}_{2} \mathrm{O}$ in $\mathrm{H}_{2} \mathrm{O}$ (final concentration $1 \mathrm{mM}$, Sigma), $1 \mu \mathrm{l}$ of $50 \mathrm{mM}$ freshly prepared TCEP (final concentration $1 \mathrm{mM}$ ) and $2.5 \mu \mathrm{l}$ of $10 \mathrm{mM}$ Tris[(1-benzyl-1H-1,2,3-triazol-4$\mathrm{yl}$ )methyl]amine (TBTA) (final concentration $500 \mu \mathrm{M})$ ) was added. The samples were rocked at room temperature for $1 \mathrm{~h}$.

\section{In-gel fluorescence profiling and western blot}


Protein pellet was precipitated by using a mixture of methanol-chloroform- $\mathrm{H}_{2} \mathrm{O}(4: 1.5: 3$, relative to sample volume). After mixing by inversion several times, samples were centrifuged at $20,000 \mathrm{xg}$ for $5 \mathrm{~min}$ at $4^{\circ} \mathrm{C}$. Two separate phases were observed with a protein pellet between the two. After carefully removing the aqueous (top) layer, $1 \mathrm{~mL}$ of prechilled methanol was added and centrifuged. After another wash with methanol, the protein pellet was dried using speed-vacuum for $10 \mathrm{~min}$. The pellet was resuspended in 1X Laemmli sample buffer $(20 \mu \mathrm{l})$ and was heated for $10 \mathrm{~min}$ at $95^{\circ} \mathrm{C}$ and separated by gel electrophoresis. In-gel fluorescence scanning was performed using a Bio-Rad ChemiDoc MP Imaging System. Gels were transferred to nitrocellulose membranes using BioRad Trans-Blot Semi-Dry Cell ( $20 \mathrm{~V}, 40 \mathrm{~min}$ ), which were blocked with PBST (0.05\% Tween-20 in PBS) containing 5\% nonfat milk for $1 \mathrm{~h}$ at room temperature. The membranes were then incubated with primary antibodies conjugated to HRPovernight at $4{ }^{\circ} \mathrm{C}$. After overnight incubation, membranes were washed with PBST three times and further incubated with secondary antibodies conjugated to HRP at room temperature if needed and developed using Bio-Rad Clarity Western ECL substrate and imaged with a Bio-Rad ChemiDoc MP Imager. Quantification of band intensities in fluorescence gels (top band) and Western blotting were performed with Image Lab (Bio-Rad). Fluorescence signal normalized to IFITM3 protein levels for three biological replicates were quantified and averaged for plotting.

\section{Influenza A virus infection assay}

For the infection assay, HEK293T cells were transfected and treated as described above to express chemically lipidated IFITM3. Then the cells were infected with influenza virus $\mathrm{A} / \mathrm{PR} / 8 / 34$ virus $(\mathrm{H} 1 \mathrm{~N} 1)$ with $\mathrm{MOI}$ of 2.5 . After $6 \mathrm{~h}$, cells were washed with PBS, trypsinized, and collected in cluster tubes. Cells were washed again with PBS and then fixed with $240 \mu \mathrm{l}$ of $4 \%$ PFA in PBS for 5 min. The fixed cells were permeabilized with $200 \mu \mathrm{l}$ of $0.2 \%$ saponin in PBS for 10 min and then blocked with 200 $\mu \mathrm{L}$ of $0.2 \% \mathrm{BSA}$ and $0.2 \%$ saponin in PBS for $10 \mathrm{~min}$. Cells were treated with antiinfluenza NP antibody conjugated to AlexaFluor-647 (1:250) and anti-HA antibody conjugated to AlexaFluor-594 (1:250) in $0.02 \%$ saponin in PBS. After three washes with $0.02 \%$ saponin in PBS, cells were resuspended in $100 \mu \mathrm{L}$ PBS containing $0.2 \%$ BSA and $0.02 \%$ saponin. The samples were analyzed by flow cytometry (BD LSRII). Data analysis was performed using FlowJo software. All samples were first gated by IFITM3positive staining, indicating successful transfection, and then NP-positive percentage of NP-positive staining, indicated successful infection.

\section{Molecular dynamics simulations}

All Molecular dynamics simulation systems were prepared using the CHARMM36 force field for protein and lipid ${ }^{3-5}$. The initial Apo IFITM3 structure was built using IFITM3 sequence data ${ }^{6}$ and typical $\alpha$-helical $\phi / \psi$ angles $\left(-57.8^{\circ}\right.$ for $\phi$ and $-47.0^{\circ}$ for $\psi$ ) for $\mathrm{AH} 1$, $\mathrm{AH} 2$, and TM helices. The initial structure was then equilibrated in an implicit solvent (GBSW) environment with $\mathrm{CHARMM}^{3,7,8}$. The positional restraints were applied during the equilibration to place TM in membrane region and $\mathrm{AH} 1$ and $\mathrm{AH} 2$ on the membrane surface. After the equilibration, three simulation systems (Apo, Cys72-Palm, and Cys72$\mathrm{mPalm}$ ) were prepared using CHARMM-GUI ${ }^{9}$ Membrane Builder ${ }^{10,11}$. The palmitoyl group was added at Cys72 during the Membrane Builder system building process for 
Cys72-Palm system. The maleimide group in maleimide-palmitate was parameterized by analogy of C3/C5 dyes in CHARMM-GUI ${ }^{12}$ and added to the IFITM3 structure for Cys72-mPalm system. TIP3P water ${ }^{13}$ and $150 \mathrm{mM} \mathrm{KCl}$ ion were added to the bulk region with for the ionic strength of cellular environment. The upper and lower leaflet contains 94 number of DMPC lipids with the box size of $80 \times 80 \times 100 \AA^{3}$. Equilibration of system were conducted with CHARMM-GUI standard protocol, and the productions were simulated with OpenMM 7.114. The particle-mesh Ewald method ${ }^{15}$ was used for long-range electrostatic interaction, and the SHAKE algorithm ${ }^{16}$ was utilized for fixing all bonds including hydrogen atom. The temperature of each systems was held at $308.15 \mathrm{~K}$ using Langevin dynamics ${ }^{17}$, and pressure was stayed at 1 bar under the semi-isotropic Monte-Carlo barostat method ${ }^{18,19}$ with a 5 ps $^{-1}$ coupling frequency. A force-based switching method ${ }^{20}$ was used for the van der Waals interactions under 10 to 12 angstrom cut-off range. To reduce the uncertainty of sequence-based structure, $1.5 \mu \mathrm{s}$ of preliminary simulation was performed and the coordinate information from that of simulation was used for the later simulation. Among $1 \mu \mathrm{s}$ of production runs, the last 500 ns trajectories were used for analysis, and each system has 3 replicas to obtain better sampling.

\section{Maleimide palmitate synthesis}

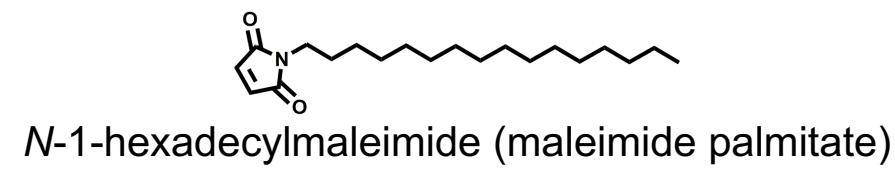

A round bottom flask was charged with $0.68 \mathrm{~g}$ of triphenylphosphine $(2.57 \mathrm{mmol}, 0.9$ eq.) and $17.5 \mathrm{ml}$ of tetrahydrofuran. The flask was placed under argon and cooled to -78 ${ }^{\circ} \mathrm{C}$. $1.18 \mathrm{ml}$ of the $40 \%$ solution of diethyl azodicarboxylate in toluene $(2.57 \mathrm{mmol}, 0.9$ eq.) were added over a period of 3 minutes. The resulting mixture was stirred for 5 minutes, after which a solution of $0.7 \mathrm{~g}$ of hexadecan-1-ol $(2.87 \mathrm{mmol}, 1 \mathrm{eq}$.$) in a$ minimal amount of THF (prepared in an argon purged vial) was added over a period of 1 minute. The resulting solution was stirred for 5 minutes. The flask's septum was then removed under the protection of an argon curtain and $0.125 \mathrm{~g}$ of neopentyl alcohol (1.43 mmol, 0.5 eq.) and $0.25 \mathrm{~g}$ of maleimide $(2.57 \mathrm{mmol}, 0.9 \mathrm{eq}$.) were added as solids. The flask was closed again under argon and the reaction mixture was stirred for 5 minutes, after which the cooling bath was removed and the reaction stirred at room temperature for 16 hours, then at $40{ }^{\circ} \mathrm{C}$ for 2 hours. After full conversion was indicated by TLC, the solvent was evaporated in vacuo. The resulting solid was purified by silica flash chromatography (loading and elution in dichloromethane). $\mathrm{N}$-1-hexadecylmaleimide was obtained after high vaccum drying $(0.481 \mathrm{~g}, 52 \%)$. $1 \mathrm{H} \mathrm{NMR}(400 \mathrm{MHz}, \mathrm{CDCl} 3) \delta \mathrm{ppm}$ 0.88 (t, J=6.59 Hz, 3H) 1.25 (br.s., 26H) $1.53-1.60(\mathrm{~m}, 2 \mathrm{H}) 3.51$ (t, J=7.23Hz, 2H) 6.68 (s,2H). 13C NMR (100MHz, CDCl3): $\delta$ ppm 14.1, 22.7, 26.7, 28.5, 29.1, 29.3, 29.5, $29.5,29.6,31.9,37.9,134.0,170.9$. This experimental procedure was adapted from Matuszak et al. ${ }^{21}$.

\section{IFITM3 purification and maleimide-palmitate coupling}


To express His $_{6}-I_{F I T M 3}$, the E. coli codon optimized gene was inserted into a pET-28c vector and transformed into BL21(DE3) E. coli, after which cells were grown in LB to 0.8 $\mathrm{OD}_{600}$ before being induced with $1 \mathrm{mM}$ IPTG overnight at $18^{\circ} \mathrm{C}$. Cells were then lysed with a probe sonicator in $2 \%$ TX-100, $25 \mathrm{mM}$ HEPES pH 7.4, $150 \mathrm{mM} \mathrm{KCl}$ and $1 \mathrm{mM}$ TCEP. After ultracentrifugation to remove insoluble cell debris $\left(30 \mathrm{~K}, 30 \mathrm{~min}, 4^{\circ} \mathrm{C}\right)$, the lysate was diluted to $<1 \% \mathrm{TX}-100$ with buffer and incubated with cobalt beads for 30 minutes at $4^{\circ} \mathrm{C}$. After washing beads with 10x CV of $0.8 \%$ TX-100, $25 \mathrm{mM} \mathrm{HEPES} \mathrm{pH}$ 7.4, $150 \mathrm{mM} \mathrm{KCl}$, and $1 \mathrm{mM}$ TCEP (Buffer A) and 10x CV Buffer A with $40 \mathrm{mM}$ imidazole, the protein was eluted with $3 x$ CV of Buffer B (Buffer A with $400 \mathrm{mM}$ Imidazole). The sample was then transferred to a $15^{\circ} \mathrm{C}$ shaker, where it was incubated with $0.5 \mathrm{mM}$ maleimide palmitate for 2 hours with a final concentration of $10 \%$ DMSO. Post incubation, the sample was diluted with Buffer A until the imidazole concentration dropped below $20 \mathrm{mM}$ and was incubated with cobalt beads for another 30 minutes. Beads were washed with 10x CV DPC buffer (0.5\% DPC, $25 \mathrm{mM} \mathrm{HEPES} \mathrm{pH} \mathrm{7.4,} 150$ $\mathrm{mM} \mathrm{KCl}$ ) before being eluted with DPC buffer $+400 \mathrm{mM}$ imidazole. In order to confirm the cysteine specificity of this reaction, n-ethyl maleimide (NEM) and $\beta$-mercaptoethanol (BME) can be used to block and quench the reaction respectively. In brief, Cys72IFITM3 was pre-incubated with $20 \mathrm{mM}$ NEM for 30 minutes prior to mPalm coupling (NEM+ condition), or mPalm was preincubated with $5 \mathrm{mM}$ BME for 30 minutes before the Cys72-IFITM3 coupling reaction (BME+ condition). Maleimide-palmitate coupling to Cys72-IFITM3 was monitored via mass shift in an Coomassie stained SDS PAGE gel.

\section{MALDI analysis}

$1 \mu \mathrm{L}$ of the sample was mixed with $9 \mu \mathrm{L}$ of matrix consisting of a saturated solution of $\alpha$ cyano-4-hydroxycinnamic acid (4-HCCA) in a 1:3:2 (v/v/v) mixture of formic acid/water/isopropanol (FWI). An aliquot of $0.5-1 \mu \mathrm{L}$ of this protein-matrix solution was spotted onto a MALDI plate precoated with an ultrathin layer of 4-HCCA matrix ${ }^{84,85}$. The sample spots were then washed for a few seconds with $2 \mu \mathrm{L}$ of cold $0.1 \%$ aqueous trifluoroacetic acid (TFA) solution. MALDI spectra were acquired in linear, delayed extraction mode using a Spiral TOF JMS-S3000 (JEOL, Tokyo, Japan). The instrument is equipped with a Nd:YLF laser, delivering $10-\mathrm{Hz}$ pulses at $349 \mathrm{~nm}$. Delayed extraction time was set at $0.75-1 \mu$ s and acquisition was performed with a sampling rate of $2 \mathrm{~ns}$. Each MALDI spectrum corresponded to an average of 1000 scans. Mass calibration was performed using horse myoglobin as protein calibrant with a technique of pseudointernal calibration wherein a few laser shots on a calibrant spot near a sample spot were collected and averaged with the sample shots into a single spectrum. The spectra were processed and analyzed using MoverZ (Proteometrics, LLC).

\section{NMR sample preparation}

To express His 6 -IFITM3, the E. coli codon optimized gene was inserted into a pET-28c vector and transformed into BL21(DE3) E. coli. For NMR experiments, cells were adapted to $100 \%$ D2O ${ }^{13} \mathrm{C}^{15} \mathrm{~N}$ M9 minimal media, before being induced with $1 \mathrm{mM}$ IPTG overnight at $18^{\circ} \mathrm{C}$. After induction, IFITM3 was purified and chemically lipidated as described above. After purification, samples were concentrated and dialyzed overnight into the final NMR buffer (0.5\% DPC, $25 \mathrm{mM}$ HEPES pH 7, $150 \mathrm{mM} \mathrm{KCl}$ ). His 6 -apo 
IFITM3 spectra were recorded on $150 \mu \mathrm{M}, 350 \mu \mathrm{M}$, and $1 \mathrm{mM}$ samples. His 6 -Cys72$\mathrm{mPalm}$ IFITM3 spectra were recorded on a sample at $350 \mu \mathrm{M}$.

\section{NMR spectroscopy and analysis}

The NMR data was acquired at $35^{\circ} \mathrm{C}(308 \mathrm{~K})$ on Bruker 800 and $900 \mathrm{MHz}$ AVANCE spectrometers equipped with $\mathrm{TCl}$ CryoProbes. The backbone resonances of ${ }^{2} \mathrm{H}^{13} \mathrm{C}^{15} \mathrm{~N}$ labeled IFITM3 with and without lipidation were assigned using a suite of multidimensional $\mathrm{N}^{15}$-TROSY based experiments: ${ }^{15} \mathrm{~N}-\mathrm{HSQC}, \mathrm{HNCA}, \mathrm{HNCO}, \mathrm{HNCACB}$, $\mathrm{HN}(\mathrm{CO}) \mathrm{CA}, \mathrm{HN}(\mathrm{CO}) \mathrm{CACB}, \mathrm{HNCACO}$ and ${ }^{15} \mathrm{~N}$-edited NOESY-HSQC. Spectra analysis was performed in CARA. The assignments were confirmed by recording 2D $\mathrm{N}^{15}$-TROSY on selectively $\mathrm{N}^{15}$-labeled samples (Ala, Ile, Val, Leu and Phe) with and without lipidation. The ${ }^{15} \mathrm{~N},{ }^{13} \mathrm{C} \alpha$, and ${ }^{13} \mathrm{C}$ ' chemical shift assignments were then used to predict the dihedral angles of the protein backbone using TALOS ${ }^{+}$. In the His 6 -apo IFITM3 sample, 70 out of 115 predicted dihedral angles were found to be "good" by TALOS+. Of the other residues, 19 residues were predicted to be dynamic ( $84 \%$ of which were in the disordered N-terminal region from residue Met1 to Val59) and 26 residues were found to be ambiguous. Out of those residues, 8 were in the structured C-terminal domain (Trp60 to Gly133) while 18 were in the disordered N-terminal region (Met1 to Val59). In the His 6 -Cys72-mPalm IFITM3 sample, 68 out of 106 predicted dihedral angles were found to be "good" by TALOS+, while 25 residues were predicted to be dynamic (21 residues in the disordered $\mathrm{N}$-terminal region, 4 residues in the structured C-terminal region). 13 residues were found to be ambiguous, with 5 in the structured Cterminal domain and 8 in the disordered $\mathrm{N}$-terminal region. For the chemical shift perturbation analysis, the chemical shift differences were calculated using a weighted average of the Euclidean distance moved:

$$
d=\sqrt{\frac{1}{2}\left[\delta_{H}^{2}+\left(0.14 \delta_{N}^{2}\right)\right]}
$$

The standard deviation of the change in chemical shift across the protein was calculated and used as a threshold value $\left(\sigma_{0}\right)$ to identify residues with significant changes in chemical shift ${ }^{22}$.

\section{Truncated construct purification}

Truncated IFITM3 constructs were inserted into a SUMO containing pET28c(+) plasmid (gift from the Lima lab at MSKCC). Lysis and His-tag purification was completed in the same manner as the full length constructs. After His purification, the samples were incubated with ULP1 $(1: 250)$ overnight at $4^{\circ} \mathrm{C}$. The next morning, the samples were incubated at $15^{\circ} \mathrm{C}$ for 2 hours with maleimide palmitate. After coupling, the samples were concentrated and buffer exchanged into 1\% OG, $25 \mathrm{mM} \mathrm{HEPES}$, and $150 \mathrm{mM} \mathrm{KCl}$ over size exclusion (SEC200).

\section{Flotation assay}

Liposomes were prepared by extruding 5mM lipid stock (80:20 POPC:Cholesterol) through a 100nm filter. As maleimide-palmitate modified 1-106 IFITM3 was unstable 
without detergent, the liposomes were mixed 1:1 with protein in $1 \%$ OG, $25 \mathrm{mM}$ HEPES, $150 \mathrm{mM} \mathrm{KCl}$ so the final protein concentration was $1 \mu \mathrm{M}$ and the detergent saturated but did not solubilize the liposomes ${ }^{23}$. The liposomes were then incubated at RT for 1 hour to promote protein incorporation, after which the liposomes were dialyzed into $25 \mathrm{mM}$ HEPES and $150 \mathrm{mM} \mathrm{KCl}$ overnight at $4^{\circ} \mathrm{C}$. Buffer was exchanged after 1 hour, then again for 2 hours the next morning. For the flotation assay, liposomes were mixed with $80 \%$ histodenz solution so the final concentration was $40 \% .600 \mu \mathrm{L}$ of this $40 \%$ histodenz solution was layered in the bottom of the ultracentrifuge tube, followed by $400 \mu \mathrm{L} \mathrm{30 \%} \mathrm{histodenz} \mathrm{and} 200 \mu \mathrm{L}$ buffer. The samples were ultracentrifuged at $150,000 \times \mathrm{g}$ for 3 hours at $4^{\circ} \mathrm{C}$, after which each sample was fractionated and acetone precipitated overnight at $-20^{\circ} \mathrm{C}$. The protein pellets were resuspended in SDS and quantified using silver stain. 


\section{REFERENCES}

(1) Shen, Y., Delaglio, F., Cornilescu, G., and Bax, A. (2009) TALOS+: a hybrid method for predicting protein backbone torsion angles from NMR chemical shifts. Journal of Biomolecular NMR 44, 213-223.

(2) Berjanskii, M. V., and Wishart, D. S. (2005) A simple method to predict protein flexibility using secondary chemical shifts. J. Am. Chem. Soc. 127, 14970-14971. (3) Brooks, B. R., Brooks, C. L., III, Mackerell, A. D., Jr., Nilsson, L., Petrella, R. J., Roux, B., Won, Y., Archontis, G., Bartels, C., Boresch, S., Caflisch, A., Caves, L., Cui, Q., Dinner, A. R., Feig, M., Fischer, S., Gao, J., Hodoscek, M., Im, W., Kuczera, K., Lazaridis, T., Ma, J., Ovchinnikov, V., Paci, E., Pastor, R. W., Post, C. B., Pu, J. Z., Schaefer, M., Tidor, B., Venable, R. M., Woodcock, H. L., Wu, X., Yang, W., York, D. M., and Karplus, M. (2009) CHARMM: The biomolecular simulation program. J. Comput. Chem. 30, 1545-1614.

(4) Klauda, J. B., Venable, R. M., Freites, J. A., O'Connor, J. W., Tobias, D. J., Mondragon-Ramirez, C., Vorobyov, I., MacKerell, A. D., and Pastor, R. W. (2010) Update of the CHARMM all-atom additive force field for lipids: validation on six lipid types. J. Phys. Chem. B 114, 7830-7843.

(5) Venable, R. M., Sodt, A. J., Rogaski, B., Rui, H., Hatcher, E., MacKerell, A. D., Jr, Pastor, R. W., and Klauda, J. B. (2014) CHARMM All-Atom Additive Force Field for Sphingomyelin: Elucidation of Hydrogen Bonding and of Positive Curvature. Biophysj 107, 134-145.

(6) Ling, S., Zhang, C., Wang, W., Cai, X., Yu, L., Wu, F., Zhang, L., and Tian, C. (2016) Combined approaches of EPR and NMR illustrate only one transmembrane helix in the human IFITM3. Sci. Rep. 1-8.

(7) Im, W., Feig, M., and Brooks, C. L., III. (2003) An Implicit Membrane Generalized Born Theory for the Study of Structure, Stability, and Interactions of Membrane Proteins. Biophysj 85, 2900-2918.

(8) Im, W., Lee, M. S., and Brooks, C. L. (2003) Generalized born model with a simple smoothing function. J. Comput. Chem. 24, 1691-1702.

(9) Jo, S., Kim, T., Iyer, V. G., and Im, W. (2008) CHARMM-GUI: A web-based graphical user interface for CHARMM. J. Comput. Chem. 29, 1859-1865.

(10) Jo, S., Lim, J. B., Klauda, J. B., and Im, W. (2009) CHARMM-GUI Membrane Builder for Mixed Bilayers and Its Application to Yeast Membranes. Biophysj 97, 50-58. (11) Wu, E. L., Cheng, X., Jo, S., Rui, H., Song, K. C., Dávila-Contreras, E. M., Qi, Y., Lee, J., Monje-Galvan, V., Venable, R. M., Klauda, J. B., and Im, W. (2014) CHARMMGUI Membrane Buildertoward realistic biological membrane simulations. J. Comput. Chem. 35, 1997-2004.

(12) Jo, S., Cheng, X., Islam, S. M., Huang, L., Rui, H., Zhu, A., Lee, H. S., Qi, Y., Han, W., Vanommeslaeghe, K., MacKerell, A. D., Roux, B., and Im, W. (2014) CHARMM-GUI PDB Manipulator for Advanced Modeling and Simulations of Proteins Containing Nonstandard Residues. Biomolecular Modelling and Simulations 1st ed., pp 235-265. Elsevier Inc.

(13) Jorgensen, W. L., Chandrasekhar, J., Madura, J. D., Impey, R. W., and Klein, M. L. (1983) Comparison of simple potential functions for simulating liquid water. The Journal of Chemical Physics 79, 926-935. 
(14) Eastman, P., Swails, J., Chodera, J. D., McGibbon, R. T., Zhao, Y., Beauchamp, K. A., Wang, L.-P., Simmonett, A. C., Harrigan, M. P., Stern, C. D., Wiewiora, R. P., Brooks, B. R., and Pande, V. S. (2017) OpenMM 7: Rapid development of high performance algorithms for molecular dynamics. PLoS Comput Biol (Gentleman, R., Ed.) 13, e1005659-17.

(15) Essmann, U., Perera, L., Berkowitz, M. L., Darden, T., Lee, H., and Pedersen, L. G. (1995) A smooth particle mesh Ewald method. The Journal of Chemical Physics 103, 8577-8593.

(16) Ryckaert, J.-P., Ciccotti, G., and Berendsen, H. J. C. (1977) Numerical Integration of the Cartesian Equations of Motion of a System with Constraints: Molecular Dynamics of n-Alkanes. J. of Comp. Phys. 1-15.

(17) Goga, N., Rzepiela, A. J., de Vries, A. H., Marrink, S. J., and Berendsen, H. J. C. (2012) Efficient Algorithms for Langevin and DPD Dynamics. J Chem Theory Comput 8 , 3637-3649.

(18) Chow, K.-H., and Ferguson, D. M. (1995) Isothermal-isobaric molecular dynamics simulations with Monte Carlo volume sampling. Computer Physics Communications 91, 283-289.

(19) Åqvist, J., Wennerström, P., Nervall, M., Bjelic, S., and Brandsdal, B. O. (2004) Molecular dynamics simulations of water and biomolecules with a Monte Carlo constant pressure algorithm. Chemical Physics Letters 384, 288-294.

(20) Steinbach, P. J., and Brooks, B. R. (1994) New spherical-cutoff methods for longrange forces in macromolecular simulation. J. Comput. Chem. 15, 667-683.

(21) Matuszak, N., Muccioli, G. G., Labar, G., and Lambert, D. M. (2009) Synthesis and in vitro evaluation of $\mathrm{N}$-substituted maleimide derivatives as selective monoglyceride lipase inhibitors. J. Med. Chem. 52, 7410-7420.

(22) Williamson, M. P. (2013) Using chemical shift perturbation to characterise ligand binding. Progress in Nuclear Magnetic Resonance Spectroscopy 73, 1-16.

(23) Rigaud, J.-L., and Lévy, D. (2003) Reconstitution of membrane proteins into liposomes. Meth. Enzymol. 372, 65-86. 